\title{
Clinical impact of prophylactic clip closure of mucosal defects after colorectal endoscopic submucosal dissection
}

\section{다(1) $(3)$}

\author{
Authors

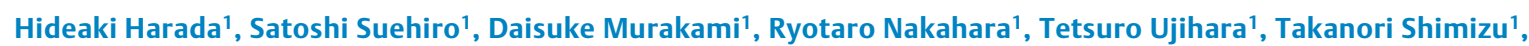 \\ Yasunaga Miyama², Yasushi Katsuyama', Kenji Hayasaka', Shigetaka Tounou
}

Institutions

1 Department of Gastroenterology, New Tokyo Hospital, Chiba, Japan

2 Department of Health Service Center, Tokyo Medical and Dental University, Tokyo, Japan

3 Second Division of Internal Medicine, Teikyo University Chiba Medical Center, Chiba, Japan

submitted 22.12.2016

accepted after revision $\quad 1.8 .2017$

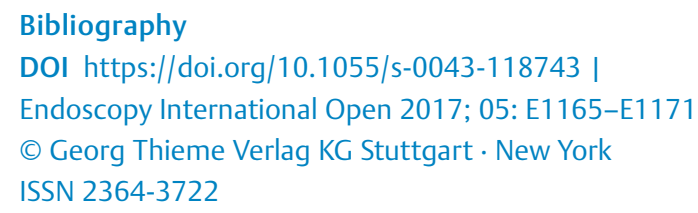

\section{Corresponding author}

Hideaki Harada, MD, Department of Gastroenterology, New Tokyo Hospital, 1271 Wanagaya, Matsudo Chiba 270-2232, Japan

Fax: +81-47-392-8718

nerimaendo@hotmail.co.jp

\section{ABSTRACT}

Background and study aims Endoscopic submucosal dissection (ESD) is useful for en bloc resection of superficial colorectal neoplasms to ensure accurate histologic diagnoses. However, colorectal ESD is associated with a high frequency of adverse events (AEs). We aimed to investigate the effectiveness of prophylactic clip closure (PCC) of mucosal defects for AEs after colorectal ESD.

Patients and methods This study included 197 patients with 211 lesions who underwent colorectal ESD between June 2010 and August 2016. Patients who had delayed perforation, delayed bleeding, abdominal pain, or fever were defined as AEs after colorectal ESD. Complete PCC was defined as completely sutured mucosal defect using endoclips following colorectal ESD, whereas incomplete PCC was defined as the mucosal defects that did not enable PCC or were partially sutured. Clinical records were retrospectively reviewed and clinical outcomes evaluated.

Results AEs occurred in 29 lesions (13.7\%), including 12 with delayed bleeding, 12 with fever, 2 with abdominal pain, 2 with fever and abdominal pain, and 1 with delayed bleeding and fever. Delayed perforation was not observed in any patient. The frequency of AEs was significantly lower in the group with complete PCC than in the group with incomplete PCC (7.3\% [9/123] vs. $22.7 \%$ [20/88]; $P<0.001)$. Multivariate analysis revealed that AEs after colorectal ESD were significantly associated with tumor size and submucosal fibrosis. Subgroup analysis among the resected specimen size of $<40 \mathrm{~mm}$ revealed that there was no significant difference in AEs between the 2 groups (5.6\% [6/107] vs. $17.8 \%$ [8/45]; $P=0.069$ ). However, the frequency of fever with complete PCC was significantly lower than that with incomplete PCC (2.8\% [3/107] vs. $13.3 \%$ [6/45]; $P=0.020)$. Conclusions Tumor size and submucosal fibrosis were independent risk factors for AEs after colorectal ESD. PCC may be effective in minimizing AEs after colorectal ESD, especially the frequency of fever.

\section{Introduction}

Endoscopic submucosal dissection (ESD) for treating superficial colorectal neoplasms allows for en bloc resection of large tumors and accurate histopathological evaluations [1-6]. However, colorectal ESD has a high adverse event (AE) frequency because of its technical difficulty and because of the thin walls, redundancy, and flexure of the colon [5,7-10]. A major $A E$ of colorectal ESD is perforation during the procedure, which can increase risk of peritonitis $[8,11-13]$. Peritonitis can be prevented in instances of minor perforation by employing endoclips to close the defect [13]. On the other hand, delayed perforation caused by transmural electrocautery burns may require emergency surgery. Moreover, transmural electrocautery burns after colorectal ESD may cause local peritonitis, resulting in abdominal pain and fever. Although these AEs can be managed by fasting and antibiotics, respectively, they result in 
longer hospitalization times and increased medical expenses [14].

Although prophylactic clip closure (PCC) of mucosal defects after colorectal endoscopic resection reduces the frequency of AEs [14 - 17]; few studies have reported the use of PCC following colorectal ESD. This study aimed to investigate the effectiveness of PCC of mucosal defects for AEs after colorectal ESD.

\section{Patients and methods}

\section{Patients}

This study was retrospective, single-center evaluation. Colorectal ESD was performed in 203 consecutive patients (217 lesions) at the New Tokyo Hospital between June 2010 and August 2016. The indication criteria for colorectal ESD were as follows; lesions with a depth of invasion limited to the mucosa or submucosa, lesions of $>20 \mathrm{~mm}$ that were difficult to resect en bloc by endoscopic mucosal resection (EMR), recurrent lesions, and residual lesions with non-lifting sign $[18,19]$. The exclusion criteria were cessation of the procedure due to severe fibrosis or difficulty in manipulating the endoscope. All patients included provided written informed consent before treatment. This study was approved by the Institutional Review Board of the New Tokyo Hospital (IRB No. NTH-0098).

\section{ESD procedure}

All colorectal lesions were initially evaluated using magnifying chromoendoscopy with crystal violet staining to determine whether ESD was indicated before treatment. ESD procedures were mainly performed by 3 ESD expert endoscopists $(\mathrm{H}$. H., S.S., and S.T.) who have performed more than 300 gastric ESDs. Antibiotics (cefmetazole $1.0 \mathrm{~g}$ ) were intravenously administered to all patients once a day before the procedure. All patients were intravenously sedated with $2 \mathrm{mg}$ midazolam doses to perform colorectal ESD under conscious sedation. Additional doses of midazolam were administered as needed. The ESD procedure was performed using a single-channel endoscope (PCF-Q260AZI or GIF-Q240; Olympus, Tokyo, Japan). Following submucosal injection with sodium hyaluronate, a distal hemicircumferential mucosal incision was made using FlushKnife BT (DK2618JB; Fujifilm, Tokyo, Japan) or Dual knife (KD-650L; Olympus, Tokyo, Japan). Next, a pocket was created in the submucosa to permit the endoscope to enter the submucosal layer for dissection. After submucosal dissection, the intact mucosa was cut using an electrosurgical knife. After tumor removal, if possible, we performed PCC. Complete PCC was defined as completely sutured the defect of the whole resection site using endoclips (ZEOCLIP ZP-CH; Zeon Medical INC., Tokyo, Japan) in a zipper fashion following colorectal ESD ( $\mathbf{F i g . 1}$ ), whereas incomplete PCC was defined as the mucosal defects that did not enable PCC or were partially sutured. If perforation occurred during colorectal ESD, the defect of the muscularis layer was closed with endoclips or the mucosal defect with perforation was completely sutured using endoclips, if possible.

The operation time was defined as the time from the submucosal injection to the completion of the operation. Submucosal fibrosis was defined as mild fibrosis or severe fibrosis on the ba- sis of endoscopic findings when sodium hyaluronate was injected into the submucosal layer.

\section{Definition of AEs}

For this study, four AEs following colorectal ESD were defined: delayed perforation, delayed bleeding, abdominal pain, and fever. Delayed perforation was defined as the presence of free or retroperitoneal air detected by abdominal radiography or abdominopelvic computed tomography after the completion of colorectal ESD if the patients had a sign of delayed perforation. Delayed bleeding was defined as overt hematochezia or melena arising from the resection site 4 hours to 28 days after the completion of colorectal ESD and/or requiring endoscopic hemostasis. Abdominal pain was defined at post-ESD clinical findings, such as sustained spontaneous pain or regional rebound tenderness, but without a frank perforation. Fever was defined as a body temperature of $\geq 37.5^{\circ} \mathrm{C}$ that developed 4 hours to 3 days after colorectal ESD.

\section{Data analysis}

Included patients were categorized into 2 groups; a complete PCC group and an incomplete PCC group. To investigate the potential risk factors for AEs after colorectal ESD, we analyzed the following factors; age ( $<70$ or $\geq 70$ years), sex, hypertension, diabetes mellitus, liver cirrhosis, renal failure, cardiovascular disease, antithrombotic agents, location of the lesion (right side of colon, left side of colon, or rectum), macroscopic type (LST-G, LST-NG, or protruded type), tumor size $(<40$ or $\geq 40 \mathrm{~mm}$ ), pathological findings (adenoma, intramucosal carcinoma, or carcinoma with submucosal invasion), fibrosis, incomplete PCC, operation time ( $<120$ or $\geq 120 \mathrm{~min}$ ), and perforation during ESD. Furthermore, we performed subgroup analysis among the resected specimen size of $<40 \mathrm{~mm}$ to investigate the effect of PCC for AEs after colorectal ESD.

\section{Statistical analysis}

Categorical variables were analyzed using the Fisher's exact test or the chi-squared test. Continuous variables were analyzed using the Mann-Whitney $U$ test. A $P$ value of $<0.05$ was considered statistically significant. Any variable found to be statistically significant in the univariate analyses was included in the multivariate logistic regression model. However, PCC was not included in the multivariate logistic regression model because of high correlation with the size of the resected specimens. All data analyses were conducted using the JMP statistical software for Windows, version 13.0 (SAS, Institute, Inc., Cary, NC, USA).

\section{Results}

\section{Baseline characteristics}

A total of 211 colorectal lesions were eligible for study inclusion. Six lesions were excluded because severe fibrosis required cessation of the procedure. Among 211 colorectal lesions, 123 lesions (58.3\%) underwent complete PCC (> Fig. 2). > Table 1 summarizes baseline characteristics and clinicopathological features of the 211 lesions. The mean age of the patients was 

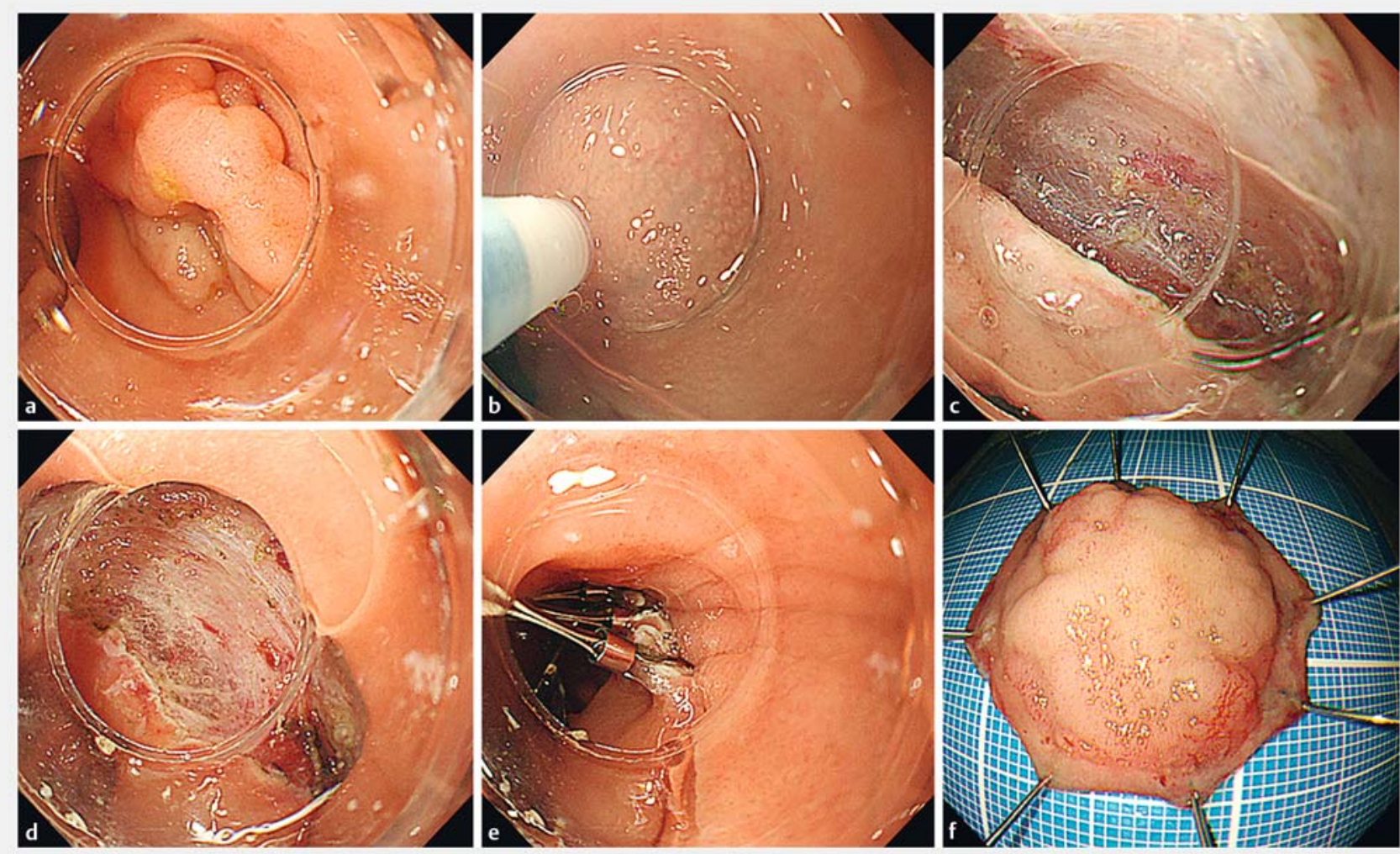

- Fig. 1 Endoscopic submucosal dissection for a superficial colorectal neoplasia. a A laterally spreading tumor was identified at the sigmoid colon (approximately $2.5 \times 2.5 \mathrm{~cm}$ ). b A mucosal incision was performed. c Submucosal dissection was performed. $\mathbf{d}$ A mucosal defect after removal of the tumor. e Complete prophylactic clip closure (PCC) was performed for the mucosal defect in a zipper fashion. $\mathbf{f}$ The specimen was resected en bloc.

Colorectal ESD for superficial neoplasms between June 2010 and August $2016(n=217)$

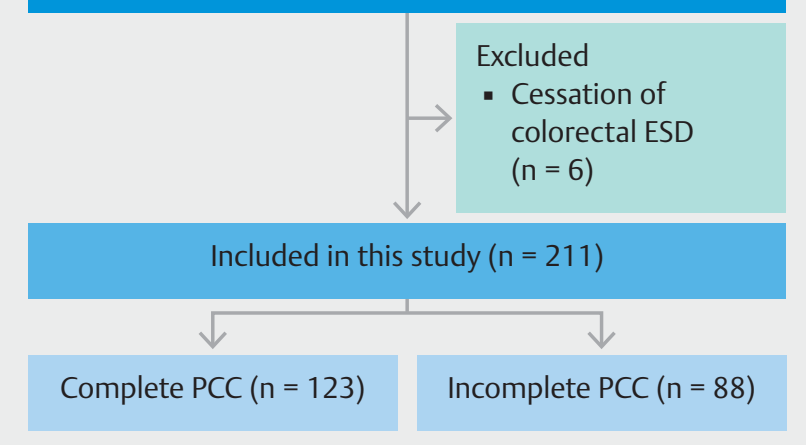

Fig. 2 Flowchart of inclusions and exclusions criteria for this study. ESD, Endoscopic submucosal dissection; PCC, prophylactic clip closure.

$70.7 \pm 9.2$ years, and 124 of 211 lesions were male (58.8\%). The median tumor size was $27 \mathrm{~mm}$, and the median operation time was $72 \mathrm{~min}$.
There were 4 perforations during colorectal ESD (1.9\% [4] 211]). Among them, the mucosal defects in 2 lesions were completely sutured using endoclips. For the other 2 lesions, although the defect of the muscularis layer due to perforation was closed with endoclips, the mucosal defects were left opened and not sutured. We categorized the former into the group with complete PCC and the latter into the group with incomplete PCC. All perforations were minor and were successfully managed conservatively.

\section{Adverse events}

AEs occurred in 29 of the 211 lesions (13.7\%). Delayed perforation was not observed in any patient. Twelve patients had delayed bleeding, 12 developed a fever, 2 had abdominal pain, 2 developed a fever and had abdominal pain, and 1 had delayed bleeding and fever ( $\triangleright$ Table 1 ). All bleeding events were successfully managed with endoscopic hemostasis, and fever and abdominal pain were successfully managed with antibiotics and fasting, respectively.

\section{Analysis of AEs}

To identify factors potentially influencing the frequency of AEs following ESD, we compared the group in which AEs occurred $(n=29)$ with the group in which no AEs occurred $(n=182)$ $(\triangleright$ Table 2). Significant differences between the two groups 
- Table 1 Baseline characteristics and clinicopathological features of the colorectal ESD.

\begin{tabular}{|c|c|}
\hline & All lesions $(n=211)$ \\
\hline Age, mean $\pm S D, y$ & $70.7 \pm 9.2$ \\
\hline Sex (male), no. (\%) & $124(58.8)$ \\
\hline Hypertension, no. (\%) & $119(56.4)$ \\
\hline Diabetes mellitus, no.(\%) & $34(16.1)$ \\
\hline Liver cirrhosis, no. (\%) & $8(3.8)$ \\
\hline Renal failure, no. (\%) & $13(6.2)$ \\
\hline Cardiovascular disease, no. (\%) & $61(28.9)$ \\
\hline Antithrombotic agents, no. (\%) & $59(28.0)$ \\
\hline \multicolumn{2}{|l|}{ Location, no. (\%) } \\
\hline - Right side of colon & $121(57.3)$ \\
\hline - Left side of colon & $47(22.3)$ \\
\hline - Rectum & $43(20.4)$ \\
\hline \multicolumn{2}{|l|}{ Macroscopic type, no. (\%) } \\
\hline - LST-G & $89(42.2)$ \\
\hline - LST-NG & $108(51.2)$ \\
\hline - Protruded type & $14(6.6)$ \\
\hline Tumor size, median (IQR), mm & $27(19-34)$ \\
\hline \multicolumn{2}{|l|}{ Pathological findings, no. (\%) } \\
\hline - Adenoma & $78(37.0)$ \\
\hline - Intramucosal carcinoma & $106(50.2)$ \\
\hline - Carcinoma with submucosal invasion & $27(12.8)$ \\
\hline Fibrosis, no. (\%) & $72(34.1)$ \\
\hline Complete PCC, no. (\%) & $123(58.3)$ \\
\hline En bloc resection, no. (\%) & $197(93.4)$ \\
\hline Operation time, median (IQR), min & $72(46-88)$ \\
\hline Hospitalization, median (IQR), days & $4.7(4-5)$ \\
\hline Perforation during ESD, no. (\%) & $4(1.9)$ \\
\hline AEs, no. (\%) & $29(13.7)$ \\
\hline - Delayed perforation, no.(\%) & $0(0)$ \\
\hline - Delayed bleeding, no. (\%) & $12(41.4)$ \\
\hline - Fever, no. (\%) & $12(41.4)$ \\
\hline - Abdominal pain, no. (\%) & $2(6.9)$ \\
\hline - Fever and abdominal pain, no. (\%) & $2(6.9)$ \\
\hline - Delayed bleeding and fever, no. (\%) & $1(3.4)$ \\
\hline \multicolumn{2}{|c|}{$\begin{array}{l}\text { ESD, endoscopic submucosal dissection; SD, standard deviation; LST-G, lat- } \\
\text { erally spreading tumors, granular type; LST-NG, laterally spreading tumors, } \\
\text { nongranular type; IQR, interquartile range; PCC, prophylactic clip closure; } \\
\text { AEs, adverse events. }\end{array}$} \\
\hline
\end{tabular}

were found with respect to tumor size $\geq 40 \mathrm{~mm}(P<0.001)$, presence of fibrosis $(P=0.005)$, incomplete PCC $(P=0.002)$, and operation time $\geq 120 \mathrm{~mm}(P=0.004)$.

Multivariate analysis with logistic regression was conducted to identify significant independent risk factors for post-ESD AEs ( $\triangleright$ Table 3). AEs had a higher likelihood of occurring in tumor size $\geq 40 \mathrm{~mm}$ (odds ratio [OR]: 3.91; $95 \%$ confidence interval $[C I]: 1.48-10.40 ; P=0.006)$ and presence of fibrosis (OR: 3.29; $95 \% \mathrm{Cl}: 1.41-7.68 ; P=0.006)$. Operation time $\geq 120 \mathrm{~min}$ was not a significant risk factor for AEs occurrence.

\section{Analysis of PCC}

Resected specimen size in the group with complete PCC had a tendency of being smaller size $(P<0.001)$. The circumference of ESD ulcers in the group with complete PCC also presented a tendency of a smaller circumference $(P<0.001)$. The frequency of AEs was significantly lower in the group with complete PCC than in the group with incomplete PCC (7.3\% [9/123] vs. $22.7 \%$ [20/88]; $P<0.001)$. There were no significant differences between the 2 groups in terms of location and operation time ( $\vee$ Table 4). The mean number of endoclips used per defect was 5.4 (range: 3 - 15). T Table 5 shows the correlation with AEs for resected specimen size $<40 \mathrm{~mm}$ between the group with complete PCC and the group with incomplete PCC. There was no significant difference in AEs between the 2 groups (5.6\% [6/ 107] vs. $17.8 \%$ [8/45]; $P=0.069$ ). Significant difference was observed between the 2 groups with regards to frequency of fever $(P=0.020)$, whereas no significant differences were observed for delayed bleeding and abdominal pain frequency ( $\downarrow$ Table 5 ).

\section{Discussion}

We investigated the effectiveness of PCC of mucosal defects for AEs after colorectal ESD. Our findings suggested that tumor size and submucosal fibrosis were independent risk factors affecting frequency of AEs. AEs such as fever and abdominal pain after colorectal ESD could be the result of bacterial invasion from the mucosal defect or transmural burn syndrome, which is similar to postpolypectomy coagulation syndrome $[20,21]$. A previous retrospective study reported that larger tumor size was significantly associated with post-ESD electrocoagulation syndrome [21]. Similarly, in this study, multivariate analysis showed that tumor size was an independent risk factor for AEs after colorectal ESD. A larger tumor size requires frequent use of electrocautery for larger submucosal dissection, resulting in transmural burn.

Fibrosis is also an independent risk factor for AEs, such as a perforation, in colorectal ESD [13, 22 -24]. In the current study, the frequency of fibrosis was significantly higher in the group with AEs than in the group without AEs. Furthermore, multivariate analysis showed that fibrosis was an independent risk factor for AEs following colorectal ESD. Frequent use of electrocautery with an electrosurgical knife due to submucosal fibrosis could cause excessive heating of the muscular layer, and transmural burn syndrome may be more likely to occur in patients with fibrosis than in those without fibrosis. 
Table 2 Comparison of the group with AEs and the group without AEs after colorectal ESD.

\begin{tabular}{|c|c|c|c|}
\hline & AEs $(n=29)$ & No AEs $(n=182)$ & $P$ value \\
\hline Age $\geq 70$ y, no. (\%) & $21(72.4)$ & $102(56.0)$ & 0.108 \\
\hline Sex (male), no. (\%) & $16(55.2)$ & $108(59.3)$ & 0.689 \\
\hline Hypertension, no. (\%) & $15(51.7)$ & $104(57.1)$ & 0.688 \\
\hline Diabetes mellitus, no. (\%) & $3(10.3)$ & $31(17.0)$ & 0.586 \\
\hline Liver cirrhosis, no. (\%) & $3(10.3)$ & $5(2.7)$ & 0.081 \\
\hline Renal failure, no. (\%) & $3(10.3)$ & $10(5.5)$ & 0.395 \\
\hline Cardiovascular disease, no. (\%) & $9(31.0)$ & $52(28.6)$ & 0.827 \\
\hline Antithrombotic agents, no. (\%) & $8(27.6)$ & $51(28.0)$ & $>0.99$ \\
\hline \multicolumn{3}{|l|}{ Location, no. (\%) } & 0.236 \\
\hline - Right side of colon & $16(55.2)$ & $105(57.7)$ & \\
\hline - Left side of colon & $4(13.8)$ & $43(22.6)$ & \\
\hline - Rectum & $9(31.0)$ & $34(18.7)$ & \\
\hline \multicolumn{3}{|l|}{ Macroscopic type, no. (\%) } & 0.478 \\
\hline " LST-G & $15(51.7)$ & $74(40.7)$ & \\
\hline . LST-NG & $12(41.4)$ & $96(52.7)$ & \\
\hline - Protruded type & $2(6.9)$ & $12(6.6)$ & \\
\hline Tumor size $\geq 40$ mm, no. (\%) & $12(41.4)$ & $21(11.5)$ & $<0.001$ \\
\hline \multicolumn{3}{|l|}{ Pathological findings, no. (\%) } & 0.142 \\
\hline - Adenoma & $9(31.0)$ & 69 (37.9) & \\
\hline - Intramucosal carcinoma & $19(65.5)$ & $87(47.8)$ & \\
\hline - Carcinoma with submucosal carcinoma & $1(3.4)$ & $26(14.3)$ & \\
\hline Fibrosis, no. (\%) & $17(58.6)$ & $55(30.2)$ & 0.005 \\
\hline Incomplete PCC, no.(\%) & $20(69.0)$ & $68(37.4)$ & 0.002 \\
\hline Operation time $\geq 120 \mathrm{~min}$, no. (\%) & $8(27.6)$ & $14(7.7)$ & 0.004 \\
\hline Perforation during ESD, no. (\%) & $2(6.9)$ & $2(1.1)$ & 0.092 \\
\hline
\end{tabular}

Table 3 Multivariate analysis with logistic regression for AEs after colorectal ESD.

\begin{tabular}{|l|l|l|}
\hline & Odds ratio $\mathbf{( 9 5} \mathbf{C C})$ & P value \\
\hline Tumor size $\geq 40 \mathrm{~mm}$ & $3.91(1.48-10.40)$ & 0.006 \\
\hline Fibrosis & $3.29(1.41-7.68)$ & 0.006 \\
\hline Operation time $\geq 120 \mathrm{~min}$ & $2.51(0.82-7.68)$ & 0.108 \\
\hline
\end{tabular}

AEs, adverse events; ESD, endoscopic submucosal dissection; $\mathrm{Cl}$, confidence interval.

Previous retrospective studies have reported that PCC of mucosal defects after EMR and ESD significantly decreased the frequency of AEs, such as fever and abdominal pain $[14,15]$. In this study, although the resected specimen size and circumfer- ence of ESD ulcers in the group with complete PCC had a tendency to be smaller than those in the group with incomplete PCC, univariate analysis of AEs in this study likewise found the frequency of AEs to be higher in the group with incomplete PCC. On the contrary, subgroup analysis of resected specimen $<40 \mathrm{~mm}$ revealed that complete PCC did not decrease the frequency of AEs. However, the frequency of fever in the group with complete PCC was significantly lower than that in the group with incomplete PCC. Moreover, although 4 minor perforations occurred during colorectal ESD in this study, AEs occurred in 2 lesions with incomplete PCC for the mucosal defect; other 2 lesions with complete PCC had no AEs. The AEs in incomplete PCC were fever, and fever and abdominal pain. Gleaned from this study, ESD with complete PCC may have been effective in minimizing effects of perforation and AEs after colorectal ESD, especially the frequency of fever. 
- Table 4 Characteristics of patients between the group with complete PCC and the group with incomplete PCC.

\begin{tabular}{|c|c|c|c|}
\hline & Complete PCC (n=123) & Incomplete PCC ( $=88)$ & $P$ value \\
\hline Location, no. (\%) & & & 0.476 \\
\hline - Right side of colon & $71(57.7)$ & $50(56.8)$ & \\
\hline - Left side of colon & $30(24.4)$ & $17(19.3)$ & \\
\hline - Rectum & $22(17.9)$ & $21(23.9)$ & \\
\hline Resected specimen size, mm, no. (\%) & & & $<0.001$ \\
\hline . $<30$ & $65(52.8)$ & $23(26.1)$ & \\
\hline - $30-39$ & $42(34.1)$ & $22(25.0)$ & \\
\hline . $40-49$ & $12(9.8)$ & $21(23.9)$ & \\
\hline . $50-59$ & $2(1.6)$ & $10(11.4)$ & \\
\hline . $60-69$ & $2(1.6)$ & $5(5.7)$ & \\
\hline . $>69$ & $0(0)$ & $7(8.0)$ & \\
\hline Circumference of ESD ulcers, no. (\%) & & & $<0.001$ \\
\hline . $<1 / 4$ & $88(71.5)$ & $29(33.0)$ & \\
\hline . $>1 / 4,<1 / 2$ & $33(26.8)$ & $49(55.7)$ & \\
\hline - $>1 / 2,<3 / 4$ & $1(0.8)$ & $10(11.4)$ & \\
\hline$\cdot>3 / 4$ & $1(0.8)$ & $0(0)$ & \\
\hline Operation time, median (IQR), min & $58(46-82)$ & $62(40-111)$ & 0.552 \\
\hline AEs, no. (\%) & $9(7.3)$ & $20(22.7)$ & $<0.001$ \\
\hline
\end{tabular}

- Table 5 The correlation with AEs for resected specimen size $<40 \mathrm{~mm}$ between the group with complete PCC and the group with incomplete PCC.

\begin{tabular}{|c|c|c|c|c|}
\hline & Complete PCC $(n=107)$ & Incomplete PCC $(n=45)$ & Odds ratio $(95 \% \mathrm{Cl})$ & $P$ value \\
\hline AEs, no. (\%) & $6(5.6)$ & $8(17.8)$ & $0.33(0.09-1.16)$ & 0.069 \\
\hline - Delayed bleeding & $3(2.8)$ & $2(4.4)$ & $0.62(0.07-7.70)$ & 0.633 \\
\hline - Abdominal pain & $1(0.9)$ & $0(0)$ & & $>0.99$ \\
\hline - Fever & $3(2.8)$ & $6(13.3)$ & $0.19(0.03-0.94)$ & 0.020 \\
\hline
\end{tabular}

Regarding to delayed bleeding, reportedly, PCC after EMR or ESD decreases it $[14,15]$. However, in this study, no significant difference was observed in the frequency of delayed bleeding in the subgroup analysis of resected specimen size of $<40 \mathrm{~mm}$ between the group with complete PCC and the group with incomplete PCC. Two patients in the group with incomplete PCC had delayed bleeding after colorectal ESD. Among them, 1 patient was receiving antithrombotic agents, and the tumor location in the other patient was adjacent to the ileocecal valve. On the other hand, 3 patients in the group with complete PCC had delayed bleeding after colorectal ESD. All of these patients were receiving antithrombotic agents. It may be difficult to compare the difference with regard to delayed bleeding between the 2 groups, because the patients with delayed bleeding had some risks for the delayed bleeding, such as receiving antithrombotic agents and tumor location.

The current study had some limitations. First, it was a retrospective review of patients treated at a single institution. Moreover, this study had a sampling bias with regard to resected specimen size and circumference of ESD ulcers between the group with complete PCC and that with incomplete PCC. Second, the sample size of the group with AEs was comparatively small. Therefore, a further prospective, randomized study will be needed in the future. 


\section{Conclusion}

In conclusion, our data suggest that tumor size and submucosal fibrosis were independent risk factors for AEs after colorectal ESD. PCC may be effective in minimizing AEs after colorectal ESD, especially the frequency of fever.

\section{Acknowledgements}

This study was reviewed and approved by the New Tokyo Hospital Institutional Review Committee. The procedure participant provided informed written consent.

\section{Competing interests}

None

\section{References}

[1] Gotoda T, Kondo H, Ono $\mathrm{H}$ et al. A new endoscopic mucosal resection procedure using an insulation-tipped electrosurgical knife for rectal flat lesions: report of two cases. Gastrointest Endosc 1999; 50: 560 563

[2] Saito Y, Uraoka T, Matsuda T et al. Endoscopic treatment of large superficial colorectal tumors: a case series of 200 endoscopic submucosal dissections (with video). Gastrointest Endosc 2007; 66: 966 973

[3] Fujishiro M, Yahagi N, Kakushima N et al. Outcomes of endoscopic submucosal dissection for colorectal epithelial neoplasms in 200 consecutive cases. Clin Gastroenterol Hepatol 2007; 5: 678-683; quiz 645

[4] Tanaka S, Oka S, Kaneko I et al. Endoscopic submucosal dissection for colorectal neoplasia: possibility of standardization. Gastrointest Endosc 2007; 66: $100-107$

[5] Saito Y, Uraoka T, Yamaguchi Y et al. A prospective, multicenter study of 1111 colorectal endoscopic submucosal dissections (with video). Gastrointest Endosc 2010; 72: 1217-1225

[6] Hotta K, Yamaguchi Y, Saito Y et al. Current opinions for endoscopic submucosal dissection for colorectal tumors from our experiences: indications, technical aspects and complications. Dig Endosc 2012; 24: $110-116$

[7] Isomoto H, Nishiyama H, Yamaguchi $\mathrm{N}$ et al. Clinicopathological factors associated with clinical outcomes of endoscopic submucosal dissection for colorectal epithelial neoplasms. Endoscopy 2009; 41: $679-683$

[8] Hayashi N, Tanaka S, Nishiyama S et al. Predictors of incomplete resection and perforation associated with endoscopic submucosal dissection for colorectal tumors. Gastrointest Endosc 2014; 79: 427 435
[9] Sato K, Ito S, Kitagawa T et al. Factors affecting the technical difficulty and clinical outcome of endoscopic submucosal dissection for colorectal tumors. Surg Endosc 2014; 28: 2959-2965

[10] Bae JH, Yang DH, Lee S et al. Optimized hybrid endoscopic submucosal dissection for colorectal tumors: a randomized controlled trial. Gastrointest Endosc 2016; 83: 584- 592

[11] Saito Y, Fukuzawa M, Matsuda T et al. Clinical outcomes of endoscopic submucosal dissection versus endoscopic mucosal resection of large colorectal tumors as determined by curative resection. Surg Endosc 2010; 24: $343-352$

[12] Tamegai Y, Saito Y, Masaki N et al. Endoscopic submucosal dissection: a safe technique for colorectal tumors. Endoscopy 2007; 39: 418 422

[13] Takamaru H, Saito Y, Yamada M et al. Clinical impact of endoscopic clip closure of perforations during endoscopic submucosal dissection for colorectal tumors. Gastrointest Endosc 2016; 84: 494- 502

[14] Zhang QS, Han B, Xu JH et al. Clip closure of defect after endoscopic resection in patients with larger colorectal tumors decreased the adverse events. Gastrointest Endosc 2015; 82: 904 - 909

[15] Fujihara S, Mori H, Kobara $\mathrm{H}$ et al. The efficacy and safety of prophylactic closure for a large mucosal defect after colorectal endoscopic submucosal dissection. Oncol Rep 2013; 30: 85-90

[16] Oka S, Tanaka S, Kanao H et al. Current status in the occurrence of postoperative bleeding, perforation and residual/local recurrence during colonoscopic treatment in Japan. Dig Endosc 2010; 22: 376 380

[17] Liaquat H, Rohn E, Rex DK. Prophylactic clip closure reduced the risk of delayed postpolypectomy hemorrhage: experience in 277 clipped large sessile or flat colorectal lesions and 247 control lesions. Gastrointest Endosc 2013; 77: 401-407

[18] Tanaka S, Oka S, Chayama K. Colorectal endoscopic submucosal dissection: present status and future perspective, including its differentiation from endoscopic mucosal resection. J. Gastroenterol 2008; 43: $641-651$

[19] Tanaka S, Tamegai Y, Tsuda S et al. Multicenter questionnaire survey on the current situation of colorectal endoscopic submucosal dissection in Japan. Dig Endosc 2010; 22: S2 - 8

[20] Yoshida N, Yagi N, Naito Y et al. Safe procedure in endoscopic submucosal dissection for colorectal tumors focused on preventing complications. World J Gastroenterol 2010; 16: 1688- 1695

[21] Jung D, Youn YH, Jahng J et al. Risk of electrocoagulation syndrome after endoscopic submucosal dissection in the colon and rectum. Endoscopy 2013; 45: $714-717$

[22] Kim ES, Cho KB, Park KS et al. Factors predictive of perforation during endoscopic submucosal dissection for the treatment of colorectal tumors. Endoscopy 2011; 43: 573-578

[23] Lee EJ, Lee JB, Choi YS et al. Clinical risk factors for perforation during endoscopic submucosal dissection (ESD) for large-sized, nonpedunculated colorectal tumors. Surg Endosc 2012; 26: 1587-1594

[24] Hong SN, Byeon JS, Lee BI et al. Prediction model and risk score for perforation in patients undergoing colorectal endoscopic submucosal dissection. Gastrointest Endosc 2016; 84: 98 - 108 